\title{
Impact of laser scanning speed on microstructure and mechanical properties of Inconel 718 alloys by selective laser melting
}

\author{
Hong-ying Wang',2, Bin-bin Wang', 'Liang Wang', Ran Cui', Liang-shun Luo', Yan-qing Su' \\ 1. National Key Laboratory for Precision Hot Processing of Metals, School of Materials Science and Engineering, Harbin Institute \\ of Technology, Harbin 150001, China \\ 2. School of Materials Science and Engineering, Jiamusi University, Jiamusi 154007, China
}

\begin{abstract}
Inconel 718 alloys were fabricated by selective laser melting under different scanning speeds to investigate the change of the morphology of molten pool, direction of grain growth, and tensile properties. Results show that as the scanning speed increases from 1,000 to $1,450 \mathrm{~mm} \cdot \mathrm{s}^{-1}$, the ratio between depth and width of molten pool increases, yet their overlapping regimes decrease. Meanwhile, increasing scanning speed can promote the solidified structure evolve from cell to columnar dendrites, and decrease the dendrite spacing from 0.54 to $0.39 \mu \mathrm{m}$; the average columnar grain size also decreases from 84.42 to $73.51 \mu \mathrm{m}$. At different scanning speeds, the preferred orientation of grains along the building is mainly $<001\rangle$ direction. In addition, the tensile properties of samples under different scanning speeds present a non-monotonic transition. The maximum ultimate tensile strength and elongation can reach 1,014 $\pm 19 \mathrm{MPa}$ and $19.04 \pm 1.12(\%)$, respectively, at the scanning speed of $1,300 \mathrm{~mm} \cdot \mathrm{s}^{-1}$.
\end{abstract}

Key words: selective laser melting (SLM); Inconel 718; molten pool; microstructure

CLC numbers: TG146.1 5 Document code: A Article ID: 1672-6421(2021)03-170-10

\section{Introduction}

Inconel 718 alloys with high strength, excellent corrosion and fatigue resistance, have received great interest in the aerospace industry ${ }^{[1,2]}$. Nevertheless, with the conventional machining method, it is difficult to manufacture Inconel 718 components with complex shapes due to the excessive tool wear and poor machinability ${ }^{[3]}$. Fortunately, selective laser melting (SLM), an additive layer-manufacturing technology, could meet the requirements for fabricating complicated geometry parts ${ }^{[4-6]}$. Using a computer-controlled high energy laser beam as the import energy source, SLM can produce dense metal parts directly from the userdefined computer aided design model by selectively fusing and consolidating pre-spread powders in a layerby-layer way ${ }^{[7-9]}$. Therefore, SLM has the capability to build any geometrically complicated components without

\section{*Liang Wang}

Male, born in 1981, Professor, doctoral supervisor. His research interests mainly focus on the melting and forming of advanced materials such as titanium alloys and superalloys. To date, he has published more than 50 papers in $\mathrm{SCl}$ indexed journals.

E-mail: wanglianghit604@163.com

Received: 2020-11-18; Accepted: 2021-03-01 any aid of tools or moulds, which need extra lead-time and investment cost ${ }^{[10,11]}$. Recently, many Ni-based high temperature components have been fabricated by $\mathrm{SLM}^{[12-15]}$. For example, Trosch et al. ${ }^{[3]}$ employed SLM technology to obtain Inconel 718 alloy successfully, whose tensile properties are better than those fabricated by casting or forging at ambient temperature, and even close to that fabricated by forging at elevated temperature.

In recent years, researchers mainly focused on the influence of process parameters on microstructure and performance ${ }^{[16-18]}$. Yi et al. ${ }^{[2]}$ reported the effect of laser energy density in the range of $0.1-0.3 \mathrm{~J} \cdot \mathrm{mm}^{-1}$ on mechanical properties, and indicated that the fewest pores and best mechanical properties were obtained at $0.2 \mathrm{~J} \cdot \mathrm{mm}^{-1}$. Jia et al. ${ }^{[16]}$ found that when increasing the applied laser energy density, the microstructure of Inconel 718 superalloy changed as follows: coarsened columnar dendrites - clustered dendrites - slender and uniformly distributed columnar dendrites. The energy density is determined by the laser power and scanning speed. The scanning speed is an important process parameter of SLM-processed Inconel 718 alloy, but the investigation of the influence of scanning speed on microstructure and mechanical properties is 
still relatively limited. Although Joon-Phil et al. ${ }^{[19]}$ gave the densification behavior and microstructure evolution of Inconel 718 alloy under different scanning speeds, there was no focus on the morphology of molten pool, or the relationship between structure and properties. As is known, a continuous molten pool is formed during SLM process. Different morphologies of molten pool determine the direction of heat flow, which affect the solidification behavior, the growth direction of the solidification microstructure, and the preferential growth of crystals. Therefore, it is necessary to conduct in-depth research.

In the present study, Inconel 718 alloy samples were fabricated by SLM with various scanning speeds. The morphology of molten pool, microstructure characteristics and crystal preferential growth at various laser scanning speeds were investigated, and the mechanical properties were assessed, aiming to establish the relationship between different

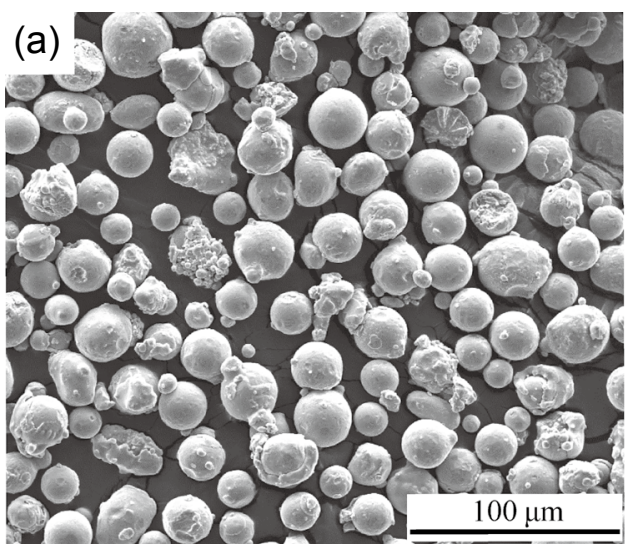

scanning speeds and molten pool morphology, microstructure characteristics, and mechanical properties of Inconel 718 parts processed by SLM.

\section{Experimental procedure}

\subsection{Sample preparation}

Inconel 718 powders were prepared by gas atomization technique. The powders are spherical particles with the diameter of 10-38 $\mu \mathrm{m}$, as shown in Fig. 1. The nominal compositions of the powders by weight are $19.2 \% \mathrm{Cr}, 17.32 \% \mathrm{Fe}$, $5.16 \% \mathrm{Nb}, 3.15 \% \mathrm{Mo}, 1.0 \% \mathrm{Ti}, 0.54 \% \mathrm{Al}, 0.23 \% \mathrm{Mn}, 0.05 \%$ $\mathrm{Co}, 0.33 \% \mathrm{Si}$, and $\mathrm{Ni}$ the balance. The powders were dried using a DZ-2BC-II drying machine to insure their good flowability.

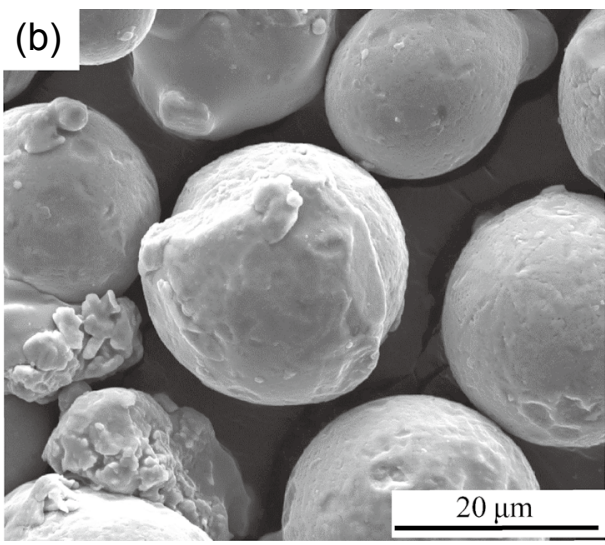

Fig. 1: Morphology of Inconel 718 powders

The printing samples were fabricated using EOS M290 SLM equipment, equipped with a continuous wavelength fiber laser, which has a spot size of $70 \mu \mathrm{m}$, maximum power of $500 \mathrm{~W}$ and wavelength of $1.06-1.10 \mu \mathrm{m}$. The 45 steel was selected as the substrate. The selected processing parameters for SLM, such as laser power, scanning speed, hatch distance, powder layer thickness and scanning pattern, are listed in Table 1 and Fig. 2. Four independent Inconel 718 alloys were prepared with different scanning speeds, marked as S1-S4.

\subsection{Experimental characterization}

Each test sample, which was segmented by wire-cutting, was firstly ground with $\mathrm{SiC}$ papers to 2,000 grit, then polished with $1 \mu \mathrm{m}$ diamond paste, and finally etched in the etchant mixture of 36vol.\% distilled water, 36vol.\% hydrochloric acid, $10 \mathrm{vol} . \%$ nitric acid, and 18vol.\% hydrogen peroxide for $2 \mathrm{~s}$. The microstructure was observed by optical microscopy (OM, Olympus GX71) and scanning electron microscopy (SEM, Quanta 200FEG). In addition, the grain morphology, grain orientation, and grain size distribution were analyzed using an SEM-based electron back scatter diffraction (EBSD) detector. The surface of EBSD samples was electrochemically polished for $10-20 \mathrm{~s}$ under an electric current of $0.75 \mathrm{~A}$ at $-25{ }^{\circ} \mathrm{C}$ in the electrolytic etchant (6\% perchloric acid, 34\% n-butyl alcohol, and $60 \%$ methyl alcohol). EBSD data was processed by TSL OIM Analysis 6.1 software. Phase analysis by X-ray diffraction (XRD) was performed using a D/max-RB X-ray powder diffractometer with $\mathrm{Cu} \mathrm{K} \alpha$ radiation at $40 \mathrm{kV}$ and $40 \mathrm{~mA}$ in

Table 1: Deposition parameters for SLM processes

\begin{tabular}{|c|c|c|c|c|c|}
\hline Sample & $\begin{array}{l}\text { Laser power } \\
\text { (W) }\end{array}$ & $\begin{array}{l}\text { Spot size } \\
(\mathrm{mm})\end{array}$ & $\begin{array}{l}\text { Scanning speed } \\
\left(\mathrm{mm} \cdot \mathrm{s}^{-1}\right)\end{array}$ & $\begin{array}{l}\text { Hatch distance } \\
(\mathrm{mm})\end{array}$ & $\begin{array}{l}\text { Layer thickness } \\
\qquad(\mathrm{mm})\end{array}$ \\
\hline S1 & \multirow{4}{*}{300} & \multirow{4}{*}{0.07} & 1,000 & \multirow{4}{*}{0.07} & \multirow{4}{*}{0.04} \\
\hline $\mathrm{S} 2$ & & & 1,150 & & \\
\hline S3 & & & 1,300 & & \\
\hline S4 & & & 1,450 & & \\
\hline
\end{tabular}




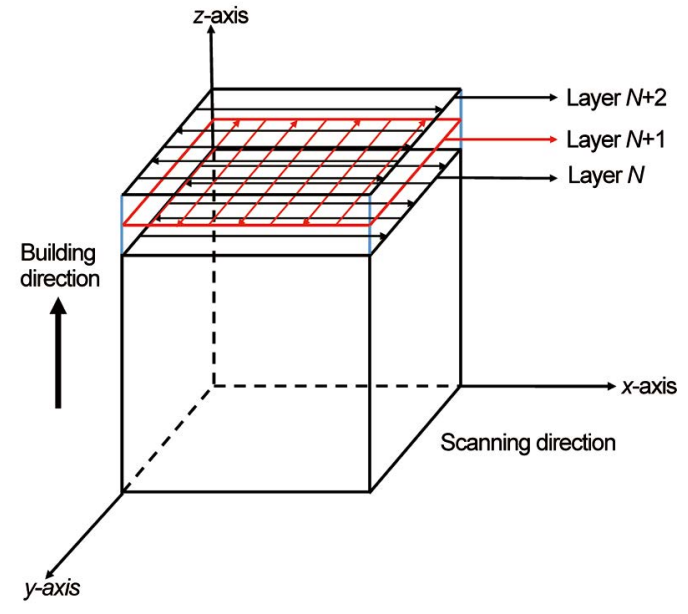

Fig. 2: Schematic of laser scanning pattern for all SLM built samples

continuous-scan mode at $5^{\circ} \cdot \mathrm{min}^{-1}$ with the range of $30^{\circ}-100^{\circ}$.

The pores of the SLM sample were observed by SEM; five images were taken at the upper, middle, lower, left and right positions in the cross section ( $y-z$ plane in Fig. 2) for each sample. The porosity is the ratio of the pore area to the total area, which can be measured and calculated by Image-ProPlus 6.0 software. Tensile tests were performed on the Instron 5569 machine with a strain rate of $5 \times 10^{4} \mathrm{~mm} \cdot \mathrm{s}^{-1}$; the dimensions for these test specimens can be found in Ref. [20]. At least three groups were tested to obtain the average value. The ductility of the material was calculated by measuring the original gauge length before and after the fracture. The elongation was the ratio of the variation of gauge length caused by plastic deformation to the original gauge length.

\subsection{Finite element analysis}

The temperature distribution in the cross-section of SLM single-track of Inconel 718 was calculated by a nonlinear heat conduction model. The selection of heat source model, the setting of initial conditions, and the calculation of the thermal variables were described in detail in Ref. [21].

\section{Results and discussion}

\subsection{Morphology and dimension of molten pool}

To study the effect of different scanning speeds on the morphology of the molten pool, the depth and width of the molten pool were measured based on the illustration in Fig. 3(a). The statistical results of the width and depth for each sample can be found in Fig. 3(b). It can be found that increasing scanning speed from 1,000 to $1,450 \mathrm{~mm} \cdot \mathrm{s}^{-1}$ simultaneously reduces the average depth (from $\sim 110$ to $88 \mu \mathrm{m}$ ) and width (from $\sim 207$ to $138 \mu \mathrm{m})$ of the molten pool.

The energy input density $E$ can be evaluated by $E=P /(v h t)^{[22]}$, where $P$ is the laser power, $v$ is the scanning speed, $h$ is the hatch distance, and $t$ is the thickness of powder layer. There is an inversely proportional relationship between $E$ and $v$, i.e., as the scanning speed increases from 1,000 to $1,450 \mathrm{~mm} \cdot \mathrm{s}^{-1}$, the energy density decreases from 107.14 to $73.89 \mathrm{~J} \cdot \mathrm{mm}^{-3}$.

A low energy density decreases the intensity of the laser radiation penetrating into the powder bed ${ }^{[2]}$, resulting in the decreased depth and width of the molten pool. In comparison with the depth, the width of the molten pool presents a stronger $v$-dependent behavior, which can be attributed to the dwell time of the laser on a fixed point. Increasing the scanning speed will reduce this dwell time, which causes the molten pool to transport less heat to the surroundings, and further reduce the width of molten pool. Therefore, it can be found that the ratio between the depth and width of molten pool synchronously rises with the scanning speed increases. As this aspect ratio is higher, a larger curvature of molten pool can be observed, which will directly affect the overlap of the molten pool. The intralayer and interlayer overlap for printing samples with different scanning speeds is shown in Fig. 4. With the increase of scanning speed, both the area for the intralayer overlap and the distance for the interlayer overlap present a reduced tendency.
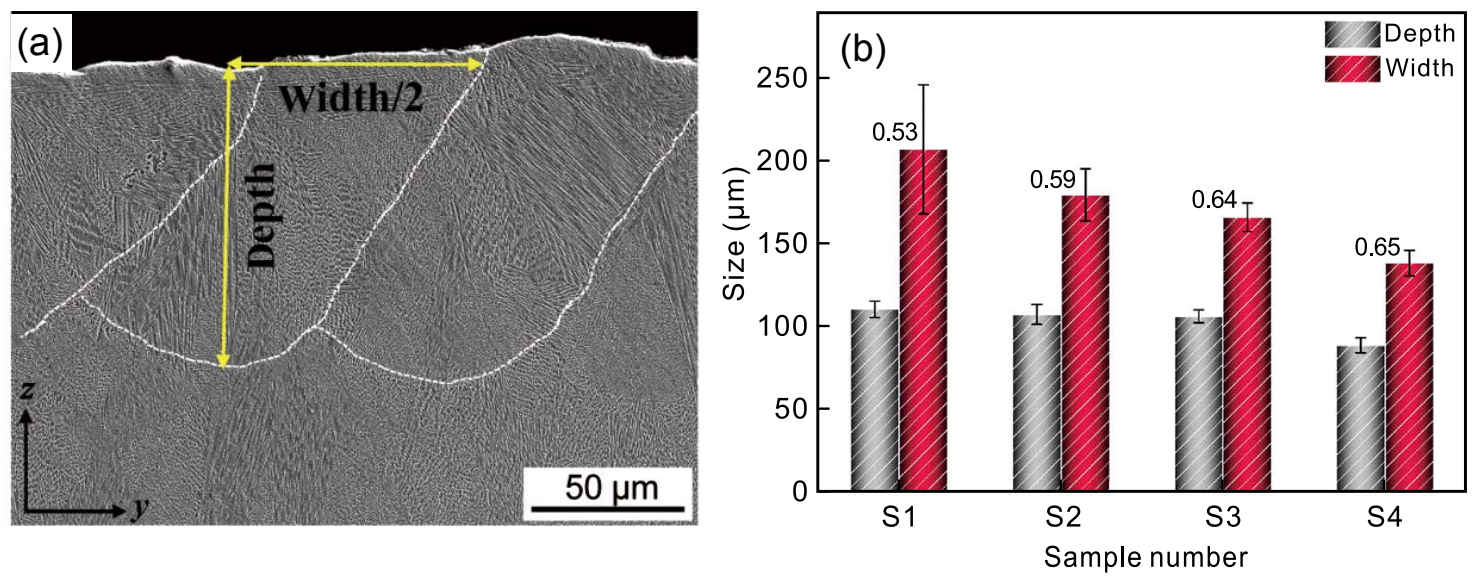

$(0.53,0.59,0.64$, and 0.65 are aspect ratio of depth/width $)$

Fig. 3: Illustration for method used to measure dimension of molten pool (a); histogram displaying depth and width of molten pool of Samples S1-S4 with different scanning speeds (b) 

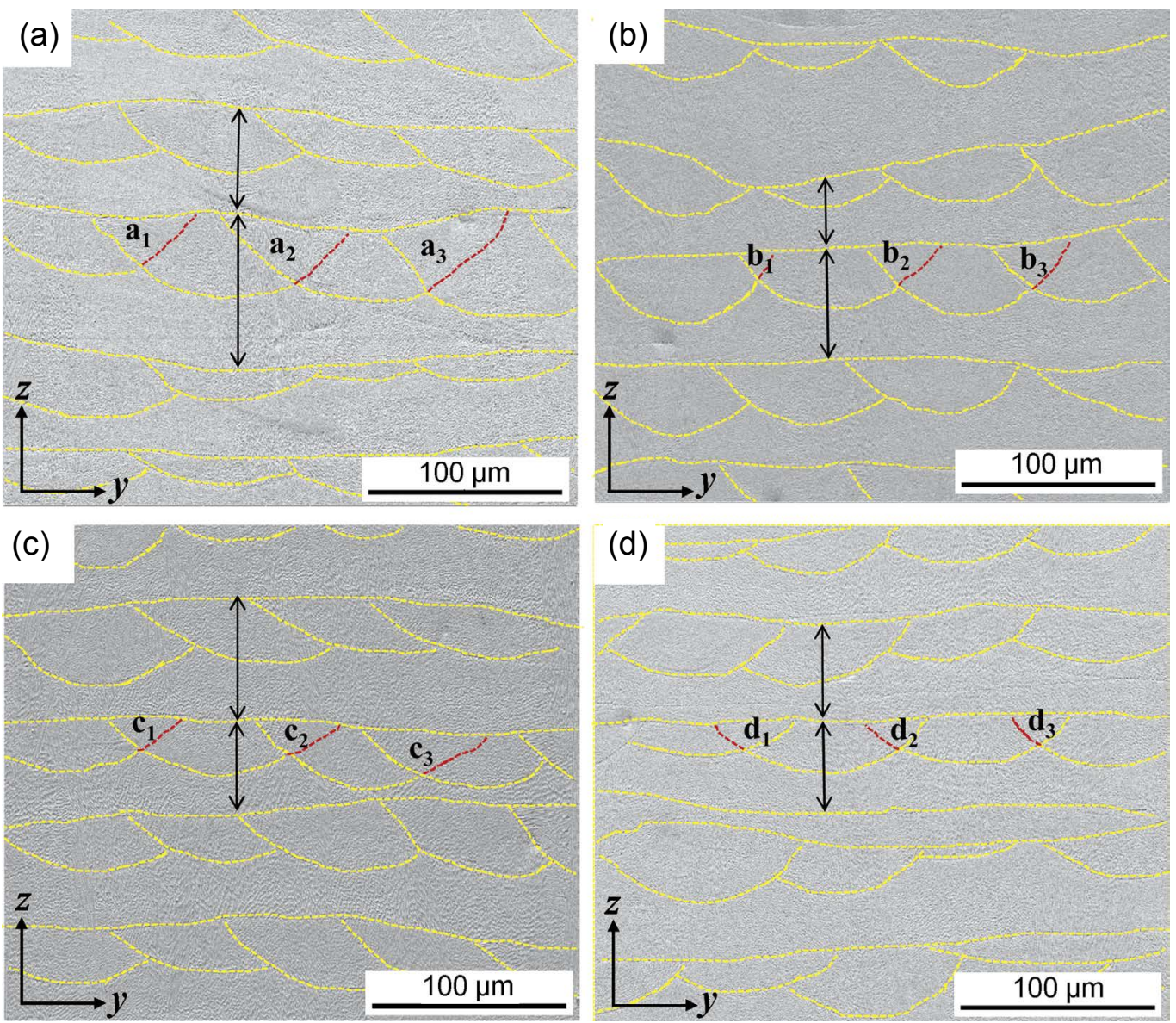

Fig. 4: Morphologies of molten pool captured from cross-section of Samples S1 (a), S2 (b), S3 (c), and S4 (d)

The yellow dotted lines delineate the boundary of molten pool, and red dotted lines is the boundary as the molten pool is unmelted. The intralayer overlapping regimes in different samples labeled as a, b, c and d, respectively. The double black arrows highlight boundary distance between different layers

\subsection{Microstructure}

Figure 5 illustrates the SEM images for the molten pool of Sample S3. Grains in Fig. 5(a) completely pass through the molten pool boundary and tend to grow in the building direction. The details can be found in Figs. 5(b) and (c). Each grain is comprised of fine cellular dendrites, and these substructures arrange in a certain direction. In addition, the growth direction of cellular dendrites at different positions (P1, P2, and P3) on the boundary of the molten pool was also measured, as shown in Fig. 5(a). The white arrows are the growth direction of cellular dendrites, and the white dotted lines indicate the direction for the maximum temperature gradient. There is an off-set angle between these two directions, whose value is respectively $7.3^{\circ}, 17.8^{\circ}$, and $13.5^{\circ}$ at P1, P2, and P3, i.e., the off-set angle near the bottom of the molten pool (P1) is smaller than that away from the bottom of the molten pool (P2 or P3). It can also be inferred that a larger aspect ratio of molten pool will lead to a greater off-set angle between the growth direction of cell dendrite and the direction of maximum temperature gradient ${ }^{[23]}$.
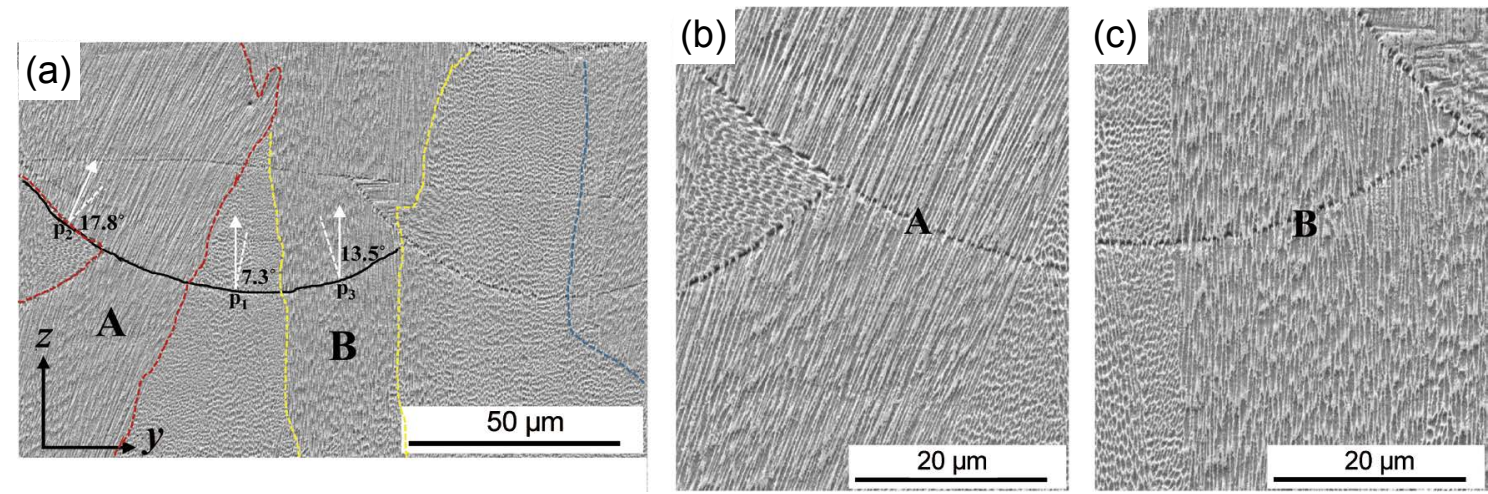

Fig. 5: (a) Scanning electron micrograph of molten pool from cross-section of Sample S3. Grains are highlighted by different colors of dotted lines. (b) and (c) are respectively details of Zones A and B in (a) 
The microstructures at the center of the molten pool in different printing samples were magnified, as shown in Fig. 6. Samples with low scanning speed (S1 and S2) present cellular dendrites structure, yet the dendrites in $\mathrm{S} 2\left(1,150 \mathrm{~mm} \cdot \mathrm{s}^{-1}\right)$ are more elongated than those in $\mathrm{S} 1\left(1,000 \mathrm{~mm} \cdot \mathrm{s}^{-1}\right)$. However, as the scanning speed continuously increases, the microstructure of S3 $\left(1,300 \mathrm{~mm} \cdot \mathrm{s}^{-1}\right)$ and $\mathrm{S} 4\left(1,450 \mathrm{~mm} \cdot \mathrm{s}^{-1}\right)$ changes to columnar dendrites, which is finer in S4. The above structural evolution mainly contributes to the solidification mode of molten pool. According to the rapid solidification theory, the increased constitutional supercooling ahead of the solidification interface may change the solidification mode from planar to cellular,
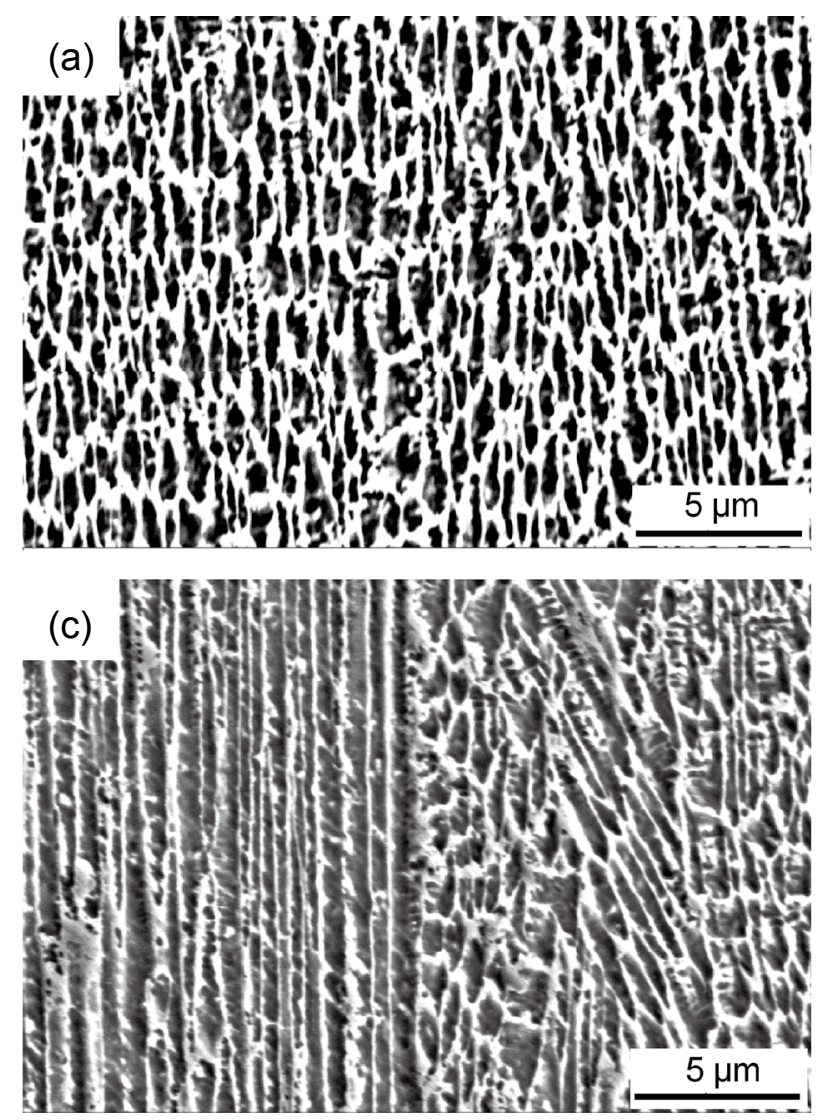

Fig. 6: Microstructures of molten pool captured from cross-section of Samples S1 (a), S2 (b), S3 (c), and S4 (d)

The primary dendrite spacing of each sample can be measured at different sites in Fig. 6 using Image6.0 software. The statistical results show that as the scanning speed increases from 1,000 to $1,450 \mathrm{~mm} \cdot \mathrm{s}^{-1}$, the primary dendrite spacing decreases from $0.54 \pm 0.07$ to $0.39 \pm 0.01 \mu \mathrm{m}$. For nickel-based alloys, based on a large number of experimental studies, the dendrite spacing $\lambda_{1}$ has a relationship with the temperature gradient $G$ and solidification rate $R$, which can be expressed as ${ }^{[25]}$ :

$$
\lambda_{1}=97(\dot{T})^{-0.36}
$$

where $\dot{T}=G \times R$ is the cooling rate. It can be seen that $\lambda_{1}$ is inversely proportional to $\dot{T}$. In this study, $\dot{T}$ was obtained by simulation calculation. With the increase of scanning speed, the maximum cooling rate of Samples $\mathrm{S} 1-\mathrm{S} 4$ are $1.57 \times 10^{7} \mathrm{~K} \cdot \mathrm{s}^{-1}$, $1.6 \times 10^{7} \mathrm{~K} \cdot \mathrm{s}^{-1}, 1.74 \times 10^{7} \mathrm{~K} \cdot \mathrm{s}^{-1}, 1.83 \times 10^{7} \mathrm{~K} \cdot \mathrm{s}^{-1}$, respectively. columnar, and even equiaxed dendrite ${ }^{[24]}$. Meanwhile, the constitutional supercooling depends on the ratio between the temperature gradient $(G)$ and solidification front rate $(R)$, where $G$ and $R$ are obtained from the finite element analysis. For Samples $\mathrm{S} 1-\mathrm{S} 4$, the maximum $G$ is $1.4 \times 10^{7} \mathrm{~K} \cdot \mathrm{m}^{-1}, 1.29 \times 10^{7} \mathrm{~K} \cdot \mathrm{m}^{-1}$, $1.24 \times 10^{7} \mathrm{~K} \cdot \mathrm{m}^{-1}$, and $1.21 \times 10^{7} \mathrm{~K} \cdot \mathrm{m}^{-1}, R$ is $1.12 \mathrm{~m} \cdot \mathrm{s}^{-1}, 1.24 \mathrm{~m} \cdot \mathrm{s}^{-1}$, $1.4 \mathrm{~m} \cdot \mathrm{s}^{-1}$, and $1.52 \mathrm{~m} \cdot \mathrm{s}^{-1}$, respectively. As the scanning speed increases, the value of $G$ decreases, but $R$ increases. As the scanning speed increases from 1,000 to $1,450 \mathrm{~mm} \cdot \mathrm{s}^{-1}$, the ratio of $G / R$ decreases from $1.25 \mathrm{~K} \cdot \mathrm{s} \cdot \mathrm{m}^{-2}$ to $0.79 \mathrm{~K} \cdot \mathrm{s} \cdot \mathrm{m}^{-2}$, which enhances the constitutional supercooling, and further alters the final solidification microstructure of printing samples.
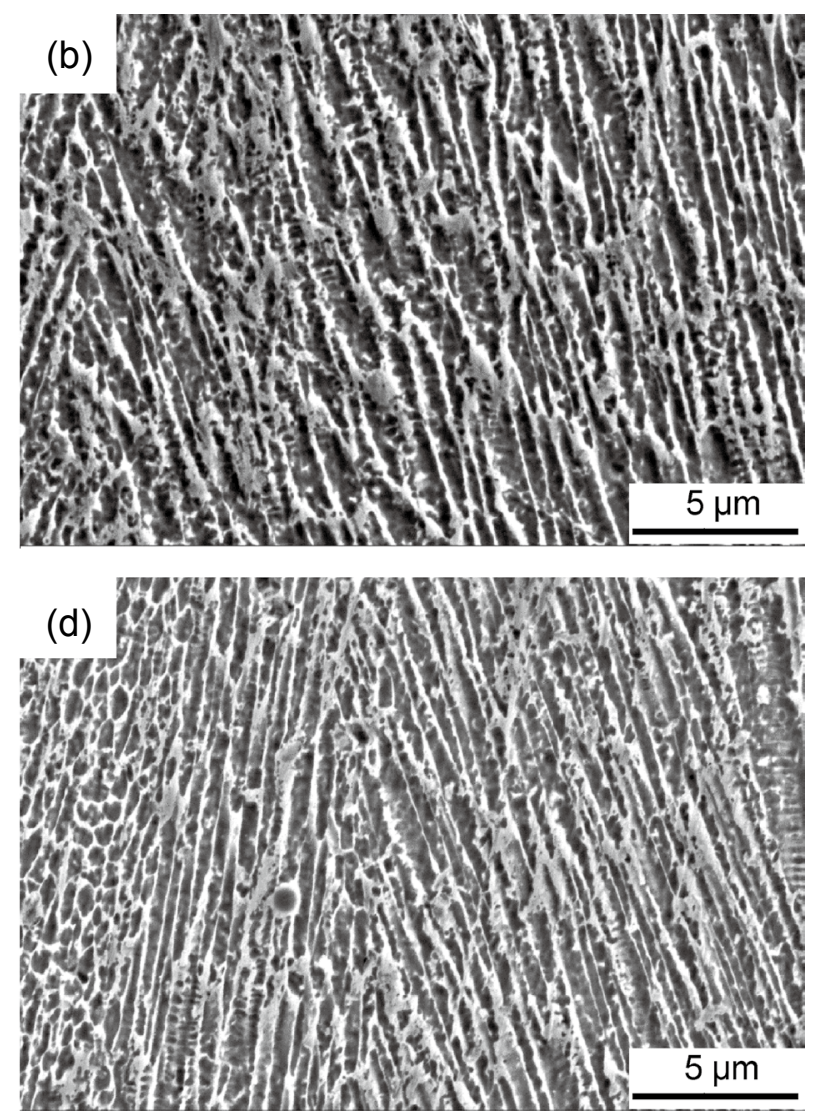

Therefore, increasing the scanning speed increases the cooling rate of printing samples, and decreases the primary dendrite spacing.

Figures 7(a)-(d) show the EBSD grain color maps at different scanning speeds. It can be seen that the microstructure in the cross-section ( $y-z$ plane) is composed of columnar grains, which tend to be parallel to the building direction ( $z$ direction). The length of the single columnar grain is about several times the width as depicted with the black dash line, as shown in Fig. 7(d). The morphology of the columnar grains mainly depends on the direction and magnitude of the temperature gradient. According to Ref. [26], the temperature gradient parallel to the building direction is greater than that parallel to the scanning direction for Inconel 718 alloy by SLM. This makes the grains grow along the building direction faster than along the scanning direction, which 

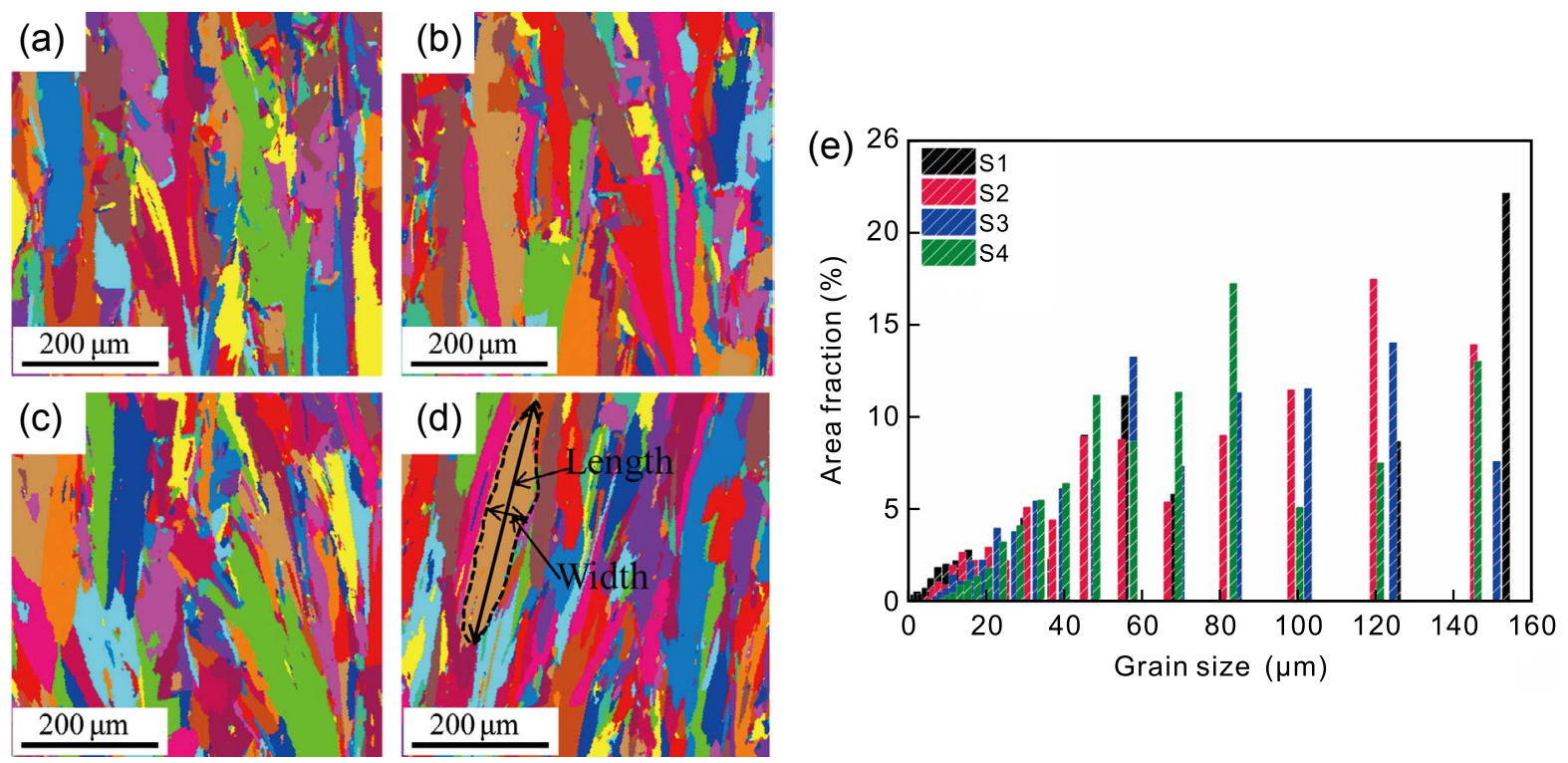

Fig. 7: EBSD maps for cross-section of Samples S1 (a), S2 (b), S3 (c), and S4 (d); and histogram showing grain size distribution (e)

causes the length of the columnar to be greater than the width To further investigate the grain size of the four samples, OIM Analysis 6.1 software was used to analyze the grain color maps of Fig. 7(a)-(d), and the obtained grain size distribution map is shown in Fig. 7(e). The grain size distributes from $3 \mu \mathrm{m}$ to 160 $\mu \mathrm{m}$ and the area fraction of grains greater than $40 \mu \mathrm{m}$ is more than $70 \%$. Among them, the maximum grain size in Sample $\mathrm{S} 1$ reaches $156 \mu \mathrm{m}$, which means this columnar grain can pass through four layers since the thickness of each layer is about 40 $\mu \mathrm{m}$. This can be attributed to the fact that the absorbed energy of powder is greater when using a slower scanning speed, resulting in the rise of the melt temperature. Thus, the final grains are coarser. The average grain size of Samples S1-S4 are $84.42 \mu \mathrm{m}$, $79.95 \mu \mathrm{m}, 77.35 \mu \mathrm{m}$ and $73.51 \mu \mathrm{m}$, respectively. That is, the average grain size decreases as the scanning speed increases. It is well known that the grain size depends on the cooling rate, which decreases as the cooling rate increases. As mentioned before, the cooling rate of Samples S1-S4 increases with the increase of the scanning speed, which causes the grains to decrease in size. In addition, because the columnar grains grow along the building direction by epitaxial grain growth, when forming the $\mathrm{N}+1$ layer, the previous $\mathrm{N}$ layer will be partially melted as the epitaxial growth substrate. The depth of the molten pool is greater with the slower scanning speed, which makes the overlapping area of the $\mathrm{N}+1$ layer and the $\mathrm{N}$ layer greater, promoting the further growth of the columnar grain. In contrast, the high scanning speed is not conducive to the growth of columnar grain.

Figures 8(a)-(d) show the grain orientation diagrams on the $y-z$ section of samples with different scanning speeds. Different colors in the figure represent different grain orientations. Compared with the triangular standard inverse pole figure, it is found that the grains in Sample S1 are almost all red, which indicates that the sample has a strong $<001>$ orientation, as shown in Fig. 8(a). Blue or green grains appear in addition to red in the other three groups of samples, indicating that a new preferred orientation appears in the building direction, such as the $<111>$ direction of Sample S2 [Fig. 8(b)], and the $<101>$ direction of Samples S3 and S4 [Figs. 8(c) and (d)]. In general, the grains in the four groups of samples still grow preferentially with a strong $<001>$ orientation. This preferential growth along the $<001>$ orientation conforms to the theory of preferential growth of cubic metals. However, as can be seen from the above, there are other preferred orientations. This is mainly because during the SLM processing, due to the continuous movement of the laser heat source, the direction of heat flow changes along the molten pool boundary. The heat flow direction changes from $90^{\circ}$ at position of the heat source to $0^{\circ}$ at the tail of the molten pool ${ }^{[27]}$. At a position far from the bottom boundary of the molten pool, the grains inclining at approximately $45^{\circ}$ with the build direction easily grow preferentially along the direction of $<101{ }^{[28]}$. However, grains that deviate from a larger angle tend to grow preferentially along the direction of $<111>$. Figure $8(\mathrm{e})$ shows the grain boundary misorientation distribution of Samples $\mathrm{S} 1-\mathrm{S} 4$. As can be seen, the grain boundary misorientation is dominated with low-angle grain boundaries (LAGBs, $<15^{\circ}$ ) and its fractions in Samples S1, S2, S3 and S4 are 59.17\%, $60.3 \%, 68.84 \%, 70.02 \%$, respectively. Due to rapid melting and solidification during the SLM process, cellular sub-grains were obtained, with LAGBs at the sub-grain boundaries. And then, LAGBs transform into high-angle grain boundaries (HAGBs, $>15^{\circ}$ ) owing to subsequent recrystallization ${ }^{[10]}$. However, due to the high solidification rate of SLM, adequate recrystallization was inhibited. Therefore, LAGBs are dominant because LAGBs are only partially transformed into HAGBs. In addition, LAGBs of S1-S4 samples increase with the increase of scanning speed. This is because the low scanning speed leads to high energy density input, which causes recrystallization in the remelting zone. In this case, LAGBs transform into HAGBs, thus leading to the reduction of LAGBs. When the scanning speed is great, the opposite is true. 


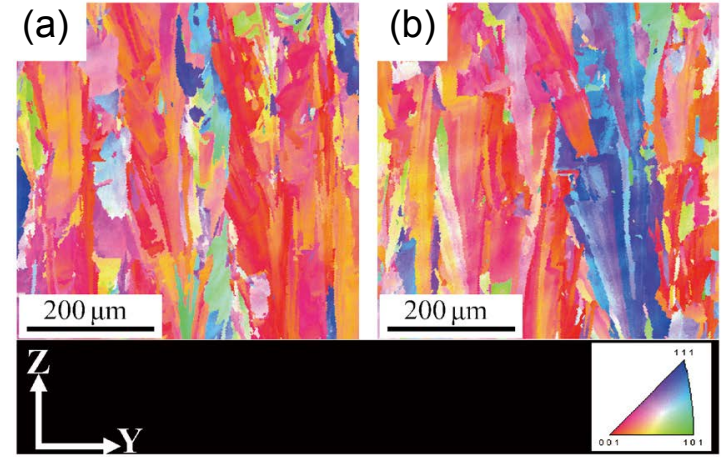

(c)

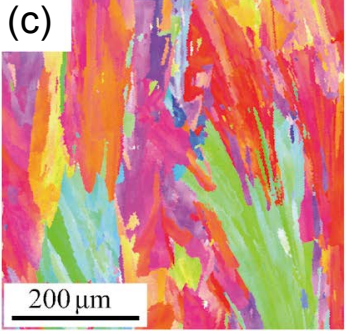

(d)

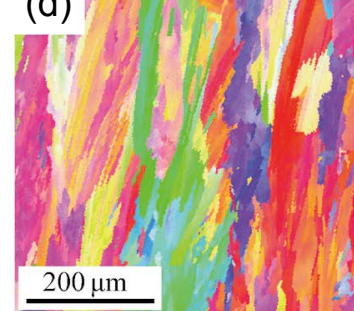

(e) 4

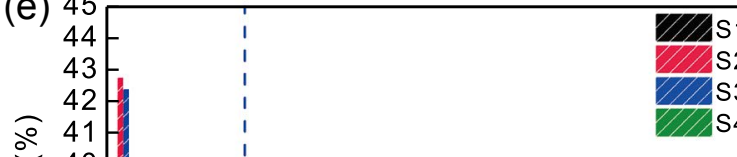

High angle grain boundaries $>15^{\circ}$

Fig. 8: EBSD IPF maps for cross-section of molten pool of Samples S1 (a), S2 (b), S3 (c), and S4 (d), and histogram showing distribution of misorientation angle of grains (e)

\subsection{Phase identification}

Figure 9 shows the X-ray diffraction patterns of Inconel 718 alloy prepared by SLM at different scanning speeds $\left(1,000-1,450 \mathrm{~mm} \cdot \mathrm{s}^{-1}\right)$. It can be seen that the strong peaks in all samples are identified as typical $\gamma$-Ni (fcc) phase, which is consistent with Ref. [29]. Among them, metallic solute atoms such as $\mathrm{Cr}, \mathrm{Nb}$ and Mo are solubilized in the $\gamma$-Ni matrix. However, the diffraction peaks of the intermetallic phases like Laves phase $\left(\mathrm{NbCr}_{2}\right), \gamma^{\prime \prime}\left(\mathrm{Ni}_{3} \mathrm{Nb}\right)$ and $\gamma^{\prime}\left[\mathrm{Ni}_{3}(\mathrm{Al}, \mathrm{Ti})\right]$ in Ref. [30] are not detected. This is because during the SLM process, the cooling rate in the molten pool is $10^{6}-10^{7} \mathrm{~K} \cdot \mathrm{s}^{-1}$. Under high cooling conditions, the solidification rate at the front of the solid-liquid interface is higher than the diffusion rate of metallic solute atoms. Therefore, at the solidification front of the solid-liquid interface, the metal solute atoms such as $\mathrm{Co}, \mathrm{Cr}$ and Mo do not have time to diffuse. They do not have much chance to form intermetallic compounds and are directly captured in the $\gamma$-Ni matrix.

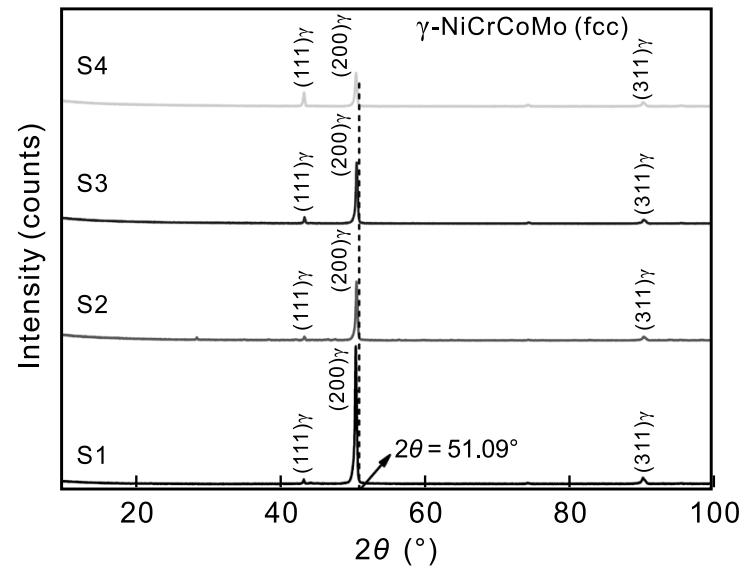

Fig. 9: XRD patterns for cross-section of SLM samples with different scanning speeds
In addition, it is also found that the diffraction peaks of the S1-S4 samples have a low-angle shift as compared to the (200) crystal plane of the standard $\gamma-\mathrm{Ni}$ phase. This offset angle increases as the scanning speed increases. This is mainly due to the different solid solubility of the alloying elements in the $\gamma$-Ni phase at different scanning speeds. At higher scanning speeds, the solidification rate at the front of the solid-liquid interface is higher due to the lower energy input. As a result, the solid solubility of alloying elements in the $\gamma$-Ni phase is relatively greater. In contrast, the solid solubility of alloying elements in the $\gamma$-Ni phase is relatively smaller at low scanning speeds. Therefore, the deviation angle of the (200) plane diffraction peak of S4 is the largest.

\subsection{Porosity and tensile property}

The SLM alloys always have inevitable metallurgical defects, and there are pores in the samples in this study. The optical macrographs of the pores on the cross section with varying scanning speeds are shown in Fig. 10. Two typical pores are observed, one is a regular circular pore, and the other is an irregular pore. The average porosity in different samples was measured, and the results can be found in Fig. 10. When the scanning speed increases from $1,000 \mathrm{~mm} \cdot \mathrm{s}^{-1}$ to $1,450 \mathrm{~mm} \cdot \mathrm{s}^{-1}$, the average porosity is $0.06 \%, 0.08 \%, 0.09 \%, 0.13 \%$, respectively. Namely, increasing the scanning speed significantly deteriorates the compactness of alloys, especially for Sample S4. The reason for this phenomenon should be attributed to the decreased input energy following the increased scanning speed; the powders are hard to melt, which is not conducive to the overlapping of the molten pool, so that the pores are easier to form.

The tensile properties of Inconel 718 fabricated by SLM with different laser scanning speeds along the building direction at room temperature are shown in Table 2. Obviously, the tensile properties increase as the scanning speed increases from 1,000 


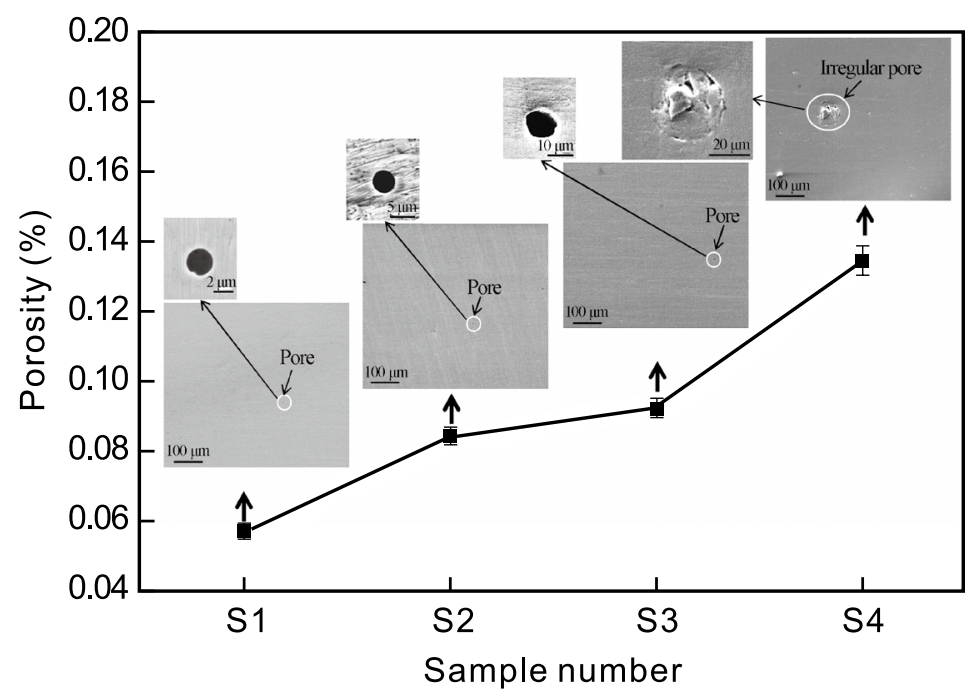

Fig. 10: Porosity for SLM samples with different scanning speeds: insets are morphologies of pores in corresponding samples $\mathrm{mm} \cdot \mathrm{s}^{-1}$ to $1,300 \mathrm{~mm} \cdot \mathrm{s}^{-1}$, and then decrease when the scanning speed further increases to $1,450 \mathrm{~mm} \cdot \mathrm{s}^{-}$ ${ }^{1}$. Among them, Sample S3 (scanning speed is 1,300 $\mathrm{mm} \cdot \mathrm{s}^{-1}$ ) has the best tensile properties, and its yield strength, ultimate tensile strength, and elongation are $752 \pm 20 \mathrm{MPa}, 1,014 \pm 19 \mathrm{MPa}, 19.04 \pm 1.12(\%)$, respectively. The strength of the as-built Inconel 718 alloy by SLM mainly depends on the width of dendrites and grain size ${ }^{[31]}$. The smaller width of dendrites and grain size will lead to better tensile properties. From the analysis of the dendrite spacing of the Samples S1-S4 in Section 3.2, it can be seen that the dendrite spacing decreases with the increase of the scanning speed. The decrease of the dendrite spacing also means the decrease of the width of the dendrite, that is, the width of the dendrite decreases as the scanning speed increases. According to the Hall-Patch relationship: $\sigma_{\mathrm{y}}=\sigma_{0}+K_{\mathrm{y}} D^{-1 / 2}$ (where $\sigma_{\mathrm{y}}$ is

Table 2: Tensile properties of Inconel 718 for different scanning speeds

$\begin{array}{cccc}\text { Sample } & \text { Yield strength (MPa) } & \text { Ultimate tensile strength }(\mathrm{MPa}) & \text { Elongation }(\%) \\ \text { S1 } & 720 \pm 7 & 957 \pm 19 & 13.97 \pm 0.77 \\ \text { S2 } & 725 \pm 25 & 992 \pm 9 & 16.54 \pm 1.15 \\ \text { S3 } & 752 \pm 20 & 1,014 \pm 19 & 19.04 \pm 1.12 \\ \text { S4 } & 703 \pm 16 & 930 \pm 23 & 10.26 \pm 0.83\end{array}$

yield strength, $\sigma_{0}$ is lattice friction, $K_{\mathrm{y}}$ is constant, $D$ is grain size), yield strength is inversely proportional to the grain size. In this study, the average grain size decreases as the scanning speed increases, so the corresponding yield strength should increase. In summary, in Samples S1-S4, the dendrite width and grain size both decrease with the increase of scanning speed. This means that the tensile strength should gradually increase, but in fact it decreases for Sample S4. This is because the tensile properties are not only related to the microstructure, but also the compactness of the component. As mentioned before, the SLM Inconel 718 alloy is not completely densified, and there are some porosities inside the specimens, which directly affect the tensile properties. The porosity in Sample $\mathrm{S} 4$ is higher than the other three samples, which leads to the reduction in tensile properties.

The fracture morphology of Inconel 718 by SLM under different scanning speeds was observed, as shown in Fig. 11. Obvious dimple features can be observed in Figs. 11 (c), (f), (i), and (1), indicating that the fracture mechanism of the four groups of samples is ductile fracture. The number of dimples in Sample S3 is significantly more than that of the other samples, which further proves that the tensile performance is better when the scanning speed is $1,300 \mathrm{~mm} \cdot \mathrm{s}^{-1}$. In the low-magnification SEM image of the fracture, it is found that there are obvious holes and lack-of-fusion defect on the fracture surfaces of the sample with the scanning speed of $1,450 \mathrm{~mm} \cdot \mathrm{s}^{-1}$. Due to the existence of these defects, stress concentration occurs near these defects during the stretching process of the specimen, which activates the generation of cracks and deteriorates the tensile properties.

\section{Conclusions}

In this study, the morphology of the melt pools, microstructure, and tensile properties of Inconel 718 alloy by SLM were studied. The main conclusions are summarized as follows:

(1) With the increase of scanning speed, the ratio between the depth and width of the molten pool increases, but the overlapping regime decreases.

(2) As the scanning speed increases from 1,000 to $1,450 \mathrm{~mm} \cdot \mathrm{s}^{-1}$, the microstructure changes from cellular to columnar dendrite, and the dendrite spacing decreases from 0.54 to $0.39 \mu \mathrm{m}$.

(3) The preferred orientation of the grains is mainly $<001>$ direction at different scanning speeds. However, due to the changes in the direction of heat flow with the increase of scanning speed, the preferred orientation of the grains is not only the $<001>$ direction, but also the $<101>$ and $<111>$ directions.

(4) As the scanning speed increases from 1,000 to $1,450 \mathrm{~mm} \cdot \mathrm{s}^{-1}$, the porosity gradually increases from $0.06 \%$ to $0.13 \%$. When the scanning speed increases to $1,300 \mathrm{~mm} \cdot \mathrm{s}^{-1}$, the ultimate tensile strength and elongation reach the maximum, which are respectively $1,014 \pm 19 \mathrm{MPa}, 19.04 \pm 1.12(\%)$. 

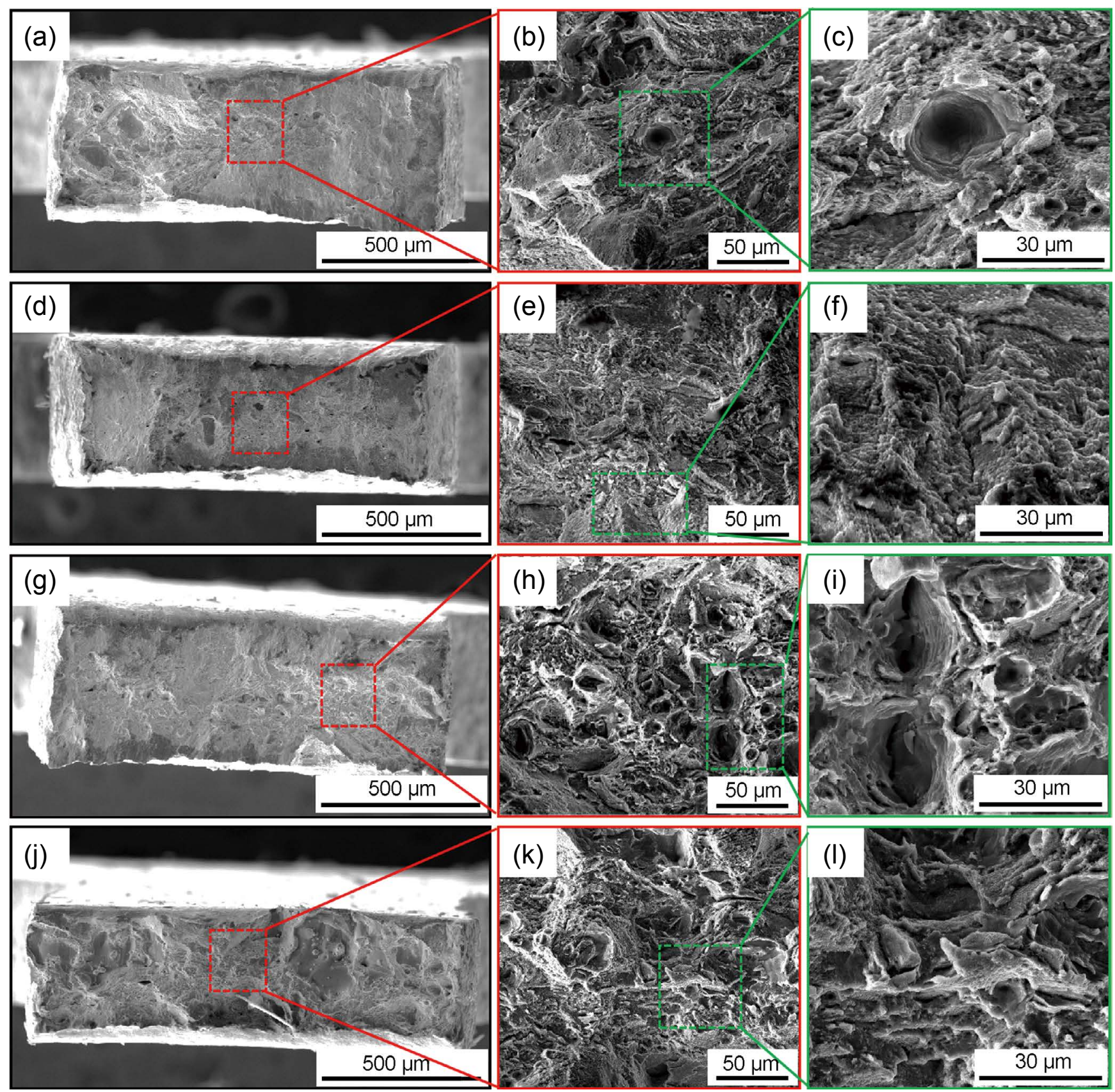

Fig. 11: Tensile fracture surfaces of Inconel 718 samples fabricated by SLM under different scanning speeds: (a-c) $1,000 \mathrm{~mm} \cdot \mathrm{s}^{-1}$ (S1); (d-f) 1,150 $\mathrm{mm} \cdot \mathrm{s}^{-1}$ (S2); (g-i) 1,300 $\mathrm{mm} \cdot \mathrm{s}^{-1}$ (S3); (j-l) 1,450 $\mathrm{mm} \cdot \mathrm{s}^{-1}$ (S4)

\section{Acknowledgements}

This work was financially supported by the National Natural Science Foundation of China (Grant Nos. 51425402 and 51501048).

\section{References}

[1] Tucho W M, Cuvillier P, Sjolyst-Kverneland A, et al. Microstructure and hardness studies of Inconel 718 manufactured by selective laser melting before and after solution heat treatment. Materials Science and Engineering: A, 2017, 689: 220-232.

[2] Yi J H, Kang J W, Wang $T \mathrm{~J}$, et al. Effect of laser energy density on the microstructure, mechanical properties, and deformation of Inconel 718 samples fabricated by selective laser melting. Journal of Alloys and Compounds, 2019, 786: 481-488.
[3] Wang Z M, Guan K, Gao M, et al. The microstructure and mechanical properties of deposited-IN718 by selective laser melting. Journal of Alloys and Compounds, 2012, 513: 518-523.

[4] Popovich V A, Borisov E V, Popovich A A, et al. Impact of heat treatment on mechanical behaviour of Inconel 718 processed with tailored microstructure by selective laser melting. Materials \& Design, 2017, 131: 12-22.

[5] Deng D, Peng R L, Brodin $\mathrm{H}$, et al. Microstructure and mechanical properties of Inconel 718 produced by selective laser melting: sample orientation dependence and effects of post heat treatments. Materials Science and Engineering: A, 2018, 713: 294-306

[6] Li J, Zhao Z Y, Bai P K, et al. Microstructural evolution and mechanical properties of IN718 alloy fabricated by selective laser melting following different heat treatments. Journal of Alloys and Compounds, 2019, 772: 861-870. 
[7] Suryawanshi J, Prashanth K G, Ramamurty U. Tensile, fracture, and fatigue crack growth properties of a 3D printed maraging steel through selective laser melting. Journal of Alloys and Compounds, 2017, 725: 355-364.

[8] Li X P, Ji G, Chen Z, et al. Selective laser melting of nano$\mathrm{TiB}_{2}$ decorated AlSi10Mg alloy with high fracture strength and ductility. Acta Materialia, 2017, 129: 183-193.

[9] Li R D, Niu P D, Yuan T C, et al. Selective laser melting of an equiatomic CoCrFeMnNi high-entropy alloy: processability, non-equilibrium microstructure and mechanical property. Journal of Alloys and Compounds, 2018, 746: 125-134.

[10] Li W, Yang Y, Liu J, et al. Enhanced nanohardness and new insights into texture evolution and phase transformation of TiAl/ $\mathrm{TiB}_{2}$ in-situ metal matrix composites prepared via selective laser melting. Acta Materialia, 2017, 136: 90-104.

[11] AlMangour B, Grzesiak D, Yang J M. Scanning strategies for texture and anisotropy tailoring during selective laser melting of TiC/316L stainless steel nanocomposites. Journal of Alloys and Compounds, 2017, 728: 424-435.

[12] Amato K N, Gaytan S M, Murr L E, et al. Microstructures and mechanical behavior of Inconel 718 fabricated by selective laser melting. Acta Materialia, 2012, 60(5): 2229-2239.

[13] Kunze K, Etter T, Grässlin J, et al. Texture, anisotropy in microstructure and mechanical properties of IN738LC alloy processed by selective laser melting (SLM). Materials Science and Engineering: A, 2015, 620: 213-222.

[14] Yoo Y S J, Book T A, Sangid M D, et al. Identifying strain localization and dislocation processes in fatigued Inconel 718 manufactured from selective laser melting. Materials Science and Engineering: A, 2018, 724: 444-451.

[15] Holland S, Wang X D, Chen J, et al. Multiscale characterization of microstructures and mechanical properties of Inconel 718 fabricated by selective laser melting. Journal of Alloys and Compounds, 2019, 784: 182-194.

[16] Jia Q B, Gu D D. Selective laser melting additive manufacturing of Inconel 718 superalloy parts: densification, microstructure and properties. Journal of Alloys and Compounds, 2014, 585: 713-721.

[17] Wang $X Q$, Keven C. Effects of thermal cycles on the microstructure evolution of Inconel 718 during selective laser melting process. Additive Manufacturing, 2017, 18: 1-14.

[18] Tao P, Li H X, Huang B Y, et al. The crystal growth, intercellular spacing and microsegregation of selective laser melted Inconel 718 superalloy. Vacuum, 2019, 159: 382-390.

[19] Choi J P, Shin G H, Yang S S, et al. Densification and microstructural investigation of Inconel 718 parts fabricated by selective laser melting. Powder Technology, 2017, 310: 60-66.
[20] Yue H Y, Chen $Y$ Y, Wang X P, et al. Microstructure, texture and tensile properties of Ti-47Al-2Cr-2Nb alloy produced by selective electron beam melting. Journal of Alloys and Compounds, 2018, 766: 450-459.

[21] Wang H Y, Wang L, Cui R, et al. Differences in microstructure and nano-hardness of selective laser melted Inconel 718 single tracks under various melting modes of molten pool. Journal of Materials Research and Technology, 2020, 9(5): 10401-10410.

[22] Thijs L, Verhaeghe F, Craeghs T, et al. A study of the microstructural evolution during selective laser melting of Ti-6Al-4V. Acta Materialia, 2010, 58(9): 3303-3312.

[23] Wang D, Song C, Yang Y, et al. Investigation of crystal growth mechanism during selective laser melting and mechanical property characterization of $316 \mathrm{~L}$ stainless steel parts. Materials \& Design, 2016, 100: 291-299.

[24] Thijs L, Kempen K, Kruth J P, et al. Fine-structured aluminium products with controllable texture by selective laser melting of pre-alloyed AISi10Mg powder. Acta Materialia, 2013, 61 (5): 1809-1819.

[25] Wang X, Carter L N, Pang B, et al. Microstructure and yield strength of SLM-fabricated CM247LC Ni-Superalloy. Acta Materialia, 2017, 128: 87-95.

[26] Zhang D Y, Feng Z, Wang C J, et al. Comparison of microstructures and mechanical properties of Inconel 718 alloy processed by selective laser melting and casting. Materials Science and Engineering: A, 2018, 724: 357-367.

[27] Thijs L, Montero Sistiaga M L, Wauthle R, et al. Strong morphological and crystallographic texture and resulting yield strength anisotropy in selective laser melted tantalum. Acta Materialia, 2013, 61(12): 4657-4668.

[28] Sun S H, Hagihara K, Nakano T. Effect of scanning strategy on texture formation in $\mathrm{Ni}-25$ at.\%Mo alloys fabricated by selective laser melting. Materials \& Design, 2018, 140: 307-316.

[29] Holland S, Wang X P, Chen J, et al. Multiscale characterization of microstructures and mechanical properties of Inconel 718 fabricated by selective laser melting. Journal of Alloys and Compounds, 2019, 784: 182-194.

[30] Yang H H, Yang J J, Huang W P, et al. The printability, microstructure, crystallographic features and microhardness of selective laser melted Inconel 718 thin wall. Materials \& Design, 2018, 156: 407-418.

[31] Wan H Y, Zhou Z J, Li C P, et al. Effect of scanning strategy on mechanical properties of selective laser melted Inconel 718 . Materials Science and Engineering: A, 2019, 753: 42-48. 